\title{
Contribución tecnológica de apoyo a la integración social del adulto mayor
}

\section{Technological contribution to support the social integration of the elderly}

\author{
Manuel Bolaños González ${ }^{1}$ (D), Cesar Alberto Collazos² (D), Francisco Gutiérrez Vela ${ }^{3}$ (D), \\ Habib Fardoun 4 (D) \\ ${ }^{1}$ Universidad de Nariño, San Juan de Pasto, Colombia \\ ${ }^{2}$ Universidad del Cauca, Popayán, Colombia \\ ${ }^{3}$ Universidad de Granada, Granada, España \\ ${ }^{4}$ King Abdulaziz University, Arabia Saudi \\ mbolanos@udenar.edu.co,ccollazo@unicauca.edu.co,fgutierr@ugr.es,hfardoun@kau.edu.sa
}

(Recibido: 7 Mayo 2020; aceptado: 7 Octubre 2020; Publicado en Internet: 1 Diciembre 2020)

\begin{abstract}
Resumen. Este documento propone la aplicación del método general de construcción de teoría en disciplinas aplicadas, establecido por Swanson y Chermack, para generar estrategias orientadas a mantener activas las habilidades cognitivas y psicomotoras de los adultos mayores. Considerando que varios autores coinciden en que las personas mayores sufren de aislamiento social y tecnológico (o incluso de exclusión) debido a su condición de envejecimiento, esta situación podría agravarse ya que la tendencia de crecimiento de la población de adultos mayores presenta niveles significativos. Es importante identificar experiencias documentadas sobre el aislamiento social de las personas mayores en el contexto global y prácticas que minimicen los efectos de este fenómeno. También es posible construir un conjunto de alternativas que ayuden a mejorar dichas habilidades con la caracterización de las habilidades cognitivas y psicomotoras de los adultos mayores.
\end{abstract}

Palabras clave: Habilidades cognitivas, Habilidades psicomotoras, Aislamiento social, Aislamiento tecnológico, Adulto mayor.

\begin{abstract}
This document proposes applying the general method of theory construction in applied disciplines, established by Swanson and Chermack, to generate strategies to maintain the elderly's cognitive and psychomotor skills of the elderly. Considering that several authors agree that older people suffer from social and technological isolation (or even exclusion) due to their aging condition, this situation could worsen since the elderly population's growth trend presents significant levels. It is important to identify documented experiences on the social isolation of older people in the global context and practices that minimize the effects of this phenomenon. It is also possible to build a set of alternatives that help improve such skills with the characterization of the elderly's cognitive and psychomotor skills of the elderly.
\end{abstract}

Keywords: Cognitive abilities, Psychomotor abilities, Social isolation, Technological isolation, Elderly.

Tipo de artículo: Artículo de investigación.

\section{Introducción}

Aging, o envejecimiento en español, está considerado como un periodo en el que la persona experimenta numerosos cambios, que, si bien dependen del estilo de vida, y del sistema social y familiar, influyen continuamente en las diversas áreas de funcionamiento (del Popolo, 2001). Los estereotipos de la cultura occidental frente al envejecimiento han llevado a que las personas experimenten cierto temor frente a la llegada de esta etapa de desarrollo, tanto que pueden ocasionar episodios de depresión, incluso hasta el aislamiento social.

En este artículo se trata de identificar la problemática asociada al aislamiento social que presentan los adultos mayores por su condición de edad y rezago tecnológico (Correia et al., 2014). Por otra parte, en 2015, había 901 millones de personas de 60 años o más, que representan el $12 \%$ de la población mundial, y está creciendo a una tasa del 3,26\% por año (United Nations, 2015). En los países desarrollados el número de personas mayores alcanza el 24\% (Klimova \& Poulova, 2018). Se espera que las personas de 65 años o 
más representen al menos el 25\% de la población europea en 2020, aumentando al 40\% entre los años 20102030 (Shore et al., 2018). De acuerdo a la revisión de 2019 de las perspectivas de población mundial (United Nations, 2019), el grupo de más rápido crecimiento son las personas mayores de 65 años, por lo cual se espera que para 2050 una de cada seis personas tendrá más de 65 años, lo que representa el 16\% de la población, y se prevé que en el norte de África y Asia occidental, Asia central y meridional, Asia oriental y sudoriental, y América Latina y el Caribe esta población se duplique para 2050, y para esta misma época en Europa y América del Norte una de cada cuatro personas tendría 65 años o más.

De acuerdo a lo publicado en el libro "Envejecimiento, personas mayores y Agenda 2030 para el Desarrollo Sostenible: Perspectiva regional y de derechos humanos" (Comisión Económica para América Latina y el Caribe, 2018), en América Latina y el caribe se estima que el aumento de personas de 60 años y más será muy rápido. De acuerdo a la Figura 1, el aumento de personas mayores es de 3,5\% anual entre 2017 y 2030, y la tasa de crecimiento esperada entre 2030 y 2060 es del 5,1\% anual. Esta información es fundamental para definir y ahondar en reformas, políticas, y proyectos necesarios para alcanzar el bienestar de los adultos mayores.

\section{(En millones de personas y porcentajes)}

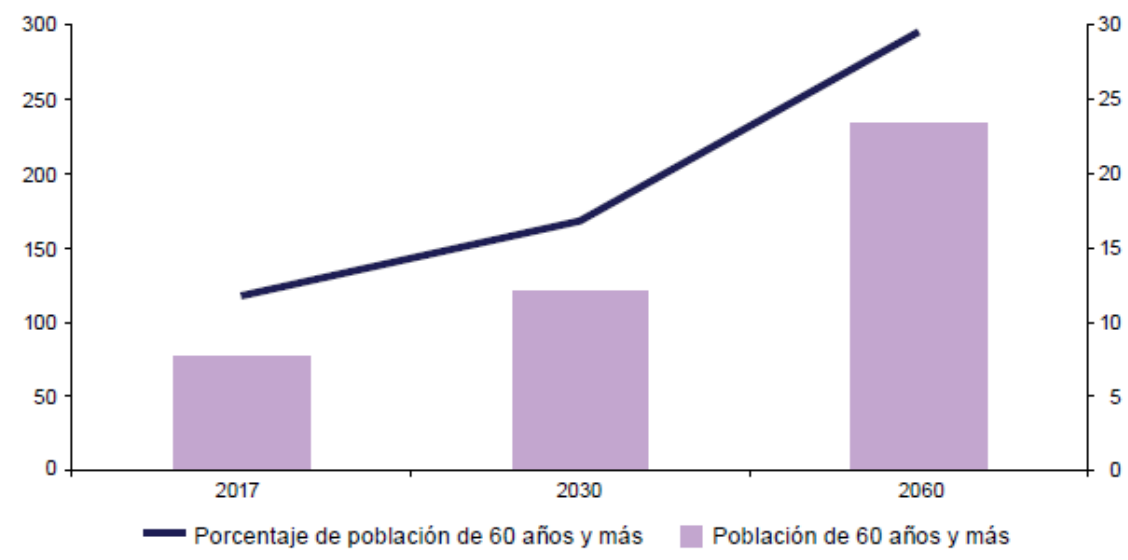

Figura 1. Número y proporción de personas de 60 años y más, 2017 - 2060 para América Latina y el Caribe Fuente: (CEPAL Desarrollo Social, 2018)

En el corto a mediano plazo, es inevitable un mayor número de personas mayores, dado que el relevo generacional está vivo. Con este panorama, la problemática de aislamiento social que tiene la población de adultos mayores se constituye en una discusión global. Lo primero es tener conciencia de que las personas mayores hacen un aporte a la sociedad, y que poseen derechos, tales como la igualdad, dignidad, el valor como persona, y libertad en el gozo de sus derechos. En este sentido, los gobiernos de todo el mundo requieren avanzar en torno a una tarea pendiente respecto del tratamiento que se debe dar a los adultos mayores frente a la globalización, los niveles de educación, y las consecuencias del cese de actividades laborales, relacionado directamente con la gestión del conocimiento de este segmento de la población.

\section{En manos de los desarrolladores}

A partir del diseño, los aspectos que deben ser tenidos en cuenta son bastante amplios. Las actuaciones del desarrollador de software para contribuir con los usuarios, en este caso específico adultos mayores, quienes utilizan la información a través de estos espacios digitales con objetivos orientados a mantener sus capacidades activas. Consecuentemente, es necesario que los desarrolladores usen recursos en Internet que son de dominio público o privado, y para estas actividades es necesario que se considere de carácter obligatorio el respeto por los materiales protegidos por la propiedad intelectual.

El desarrollador deberá tener en cuenta algunas características para entender la complejidad de las personas de edad (ver Figura 2). 


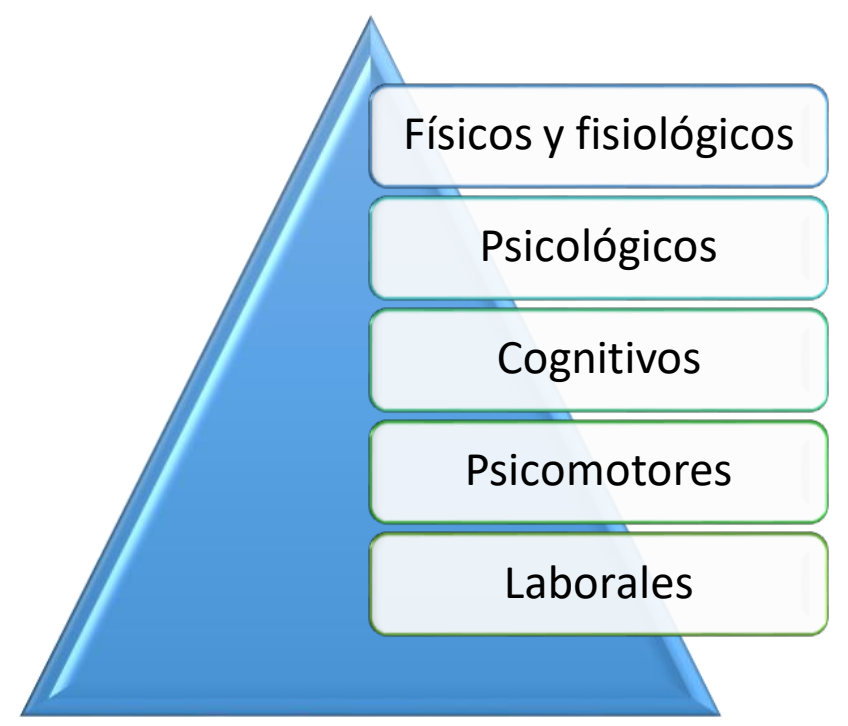

Figura 2. Cambios inherentes al proceso de envejecimiento Fuente: (Alonso Jiménez \& Moros García, 2011)

De esta manera, se debe considerar como prioritario que el envejecimiento es un proceso que no implica necesariamente la presencia de enfermedad, dependencia y disminución en la productividad (Alonso Jiménez \& Moros García, 2011). Tampoco dar por sentado condiciones en adultos mayores que estigmaticen los procesos propios software. Hay que hacer notar que se trata de considerar los avances en la materia, conocer de otros estudios, ampliar el conocimiento del estado del arte, replantear el enfoque de las investigaciones que puedan abrir nuevos horizontes en el conocimiento del cerebro del ser humano en la etapa de la involución. En particular, la Universidad de Tuebingen, Alemania, en la revista Topics in Congnitive Science hace la siguiente sugerencia frente al envejecimiento: "las funciones de nuestro cerebro se vuelven más lentas debido a que se tiene un mayor conocimiento, y no a una disminución de nuestra capacidad cognitiva" (Ramscar et al., 2014). Cabe destacar que el doctor Michael Ramscar, líder de este equipo señala que este incremento en el tiempo que le toma a nuestro cerebro el procesar una cantidad mayor de conocimiento a menudo se confunde con un deterioro cognitivo. Así pues, este dato puede significar que esta contribución tecnológica deba profundizar en este tema como posible, desde luego, es importante en el inicio colectar algunos datos personales y preferencias del usuario quien va a hacer uso del software como insumo esencial para una caracterización. Una de las formas de conocer algunos de esos aspectos que serán relevantes para la efectiva interacción en ambientes colaborativos, lúdicos y obicuos, es la adaptación de un test interactivo que permita conocer las habilidades biológicas, sicológicas y sociales, a grandes rasgos. Seguidamente se recogerán a través de diferentes instrumentos de recolección de información las preferencias en términos de temas, profesiones, tópicos de interés general, entre otros.

\section{Los usuarios: la importancia de la independencia}

Los adultos mayores se enfrentan a una serie de prejuicios que hace difícil su integración a los nuevos retos que se encuentran en la sociedad (Nazareno Boarini et al., 2006). El envejecimiento tiene lugar en el contexto social en el cual se desenvuelve el adulto mayor, y al llegar a este punto los objetivos fundamentales son tener un buen estado de salud, bienestar y tranquilidad de vida, garantizando de alguna manera estar activo física y mentalmente (Galarza Vásquez, 2012), lo cual es importante si se tiene en cuenta que uno de los principales inconvenientes de los adultos mayores es la pérdida de memoria, que implica lidiar con problemas en su desarrollo e integración social (Valencia Vásquez et al., 2011), debido a los cambios en los procesos cognitivos, aunque se debe resaltar que en las personas mayores existen notables diferencias en la manera como se presentan estos cambios, que son afectados por el entorno en el cual se viene desarrollando su vida (Nazareno Boarini et al., 2006).

Los avances tecnológicos han llevado a que se acreciente la llamada "Brecha digital", refiriéndose a las diferencias que existen entre las sociedades que tienen y las que no tienen facilidades para la utilización de 
las tecnologías de la información y las comunicaciones, todos los países no cuentan con las mismas capacidades tecnológicas que permitan a sus habitantes utilizarlas y estar a la par con otros países (Sevilla Caro et al., 2015). La Asamblea General sobre envejecimiento en el cuadragésimo sexto periodo de sesiones, en el anexo Principios de las Naciones Unidas en favor de las personas de edad, menciona en el numeral cuatro que: "las personas de edad deberán tener acceso a programas educativos y de formación adecuados" y cinco "las personas de edad deberán tener la posibilidad de vivir en entornos seguros y adaptables a sus preferencias personales y a la evolución de sus capacidades” (Naciones Unidas, 2002).

Recapitulando, se propende por la promoción del mantenimiento de las capacidades funcionales y el continuo desarrollo de habilidades cognitivas y psicomotoras en personas mayores, mediante la interacción y considerando que puede ser una contribución tecnológica para generar alternativas de participación del adulto mayor en entornos colaborativos, lúdicos, y ubicuos que permitan mantenerlas activas.

\section{Metodología basada en el diseño}

Considerando las situaciones complejas que enfrenta el adulto mayor, la contribución tecnológica tiene dos caminos por donde debe conducirse equilibradamente: en primera instancia, es necesario considerar los acuerdos existentes en materia de calidad de vida de las personas mayores (Plan de Acción Internacional de Viena sobre el Envejecimiento, Principios de las Naciones Unidas en favor de las Personas de Edad, Proclamación sobre el Envejecimiento, Declaración Política y el Plan de Acción Internacional sobre el Envejecimiento de Madrid, Segunda Asamblea Mundial sobre el Envejecimiento, entre otros (Naciones Unidas, 2002)), y en segunda instancia, se debe acomodar a una variedad de características que el adulto mayor presenta en este lapso de su vida, con el propósito de estructurar el desarrollo de la teoría de una manera consistente (Swanson \& Chermack, 2013)(ver Figura 3).

Al abordar el escenario de las implicaciones físicas, sociales, intelectuales (Menéndez Montañés \& Brochier Kist, 2011), la calidad de vida del adulto mayor se convierte en la gran meta de este planteamiento, debido a que es un segmento de la población a quien esta contribución tecnológica de apoyo a la integración social en muchas formas propende por la estimulación cognitiva, esto significa que, los entornos deberán ser adecuados a una persona que trae una experiencia, es un adulto, y que por tanto ha superado la juventud y requiere ser motivado de manera especial en esta nueva labor.

\section{The observed and experienced world}
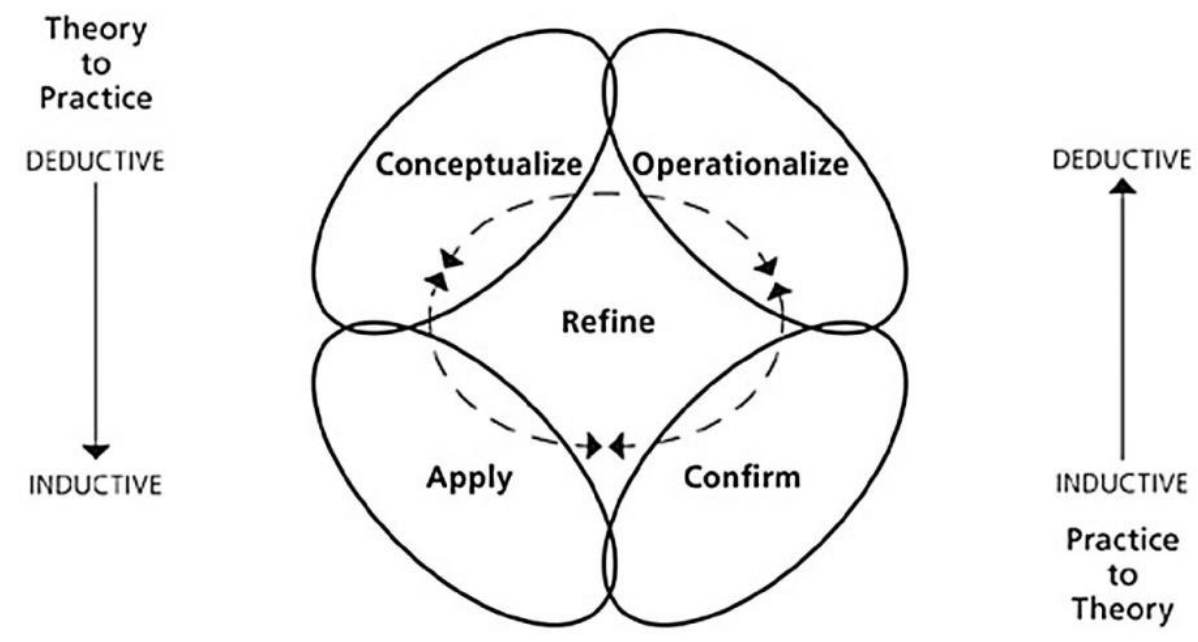

Figura 3. Método general de construcción de la teoría en las disciplinas aplicadas Fuente: (Swanson \& Chermack, 2013)

En el escenario físico y fisiológico, el procesamiento sensorial se refiere al proceso que se lleva a cabo en el Sistema Nervioso Central, que organiza la sensación del propio cuerpo con el medio ambiente y hace posible utilizar eficazmente sus facultades (Beaudry Bellefeuille, 2013), en este caso en entornos colaborativos, lúdicos y ubicuos digitales (ver Figura 4). 
En el escenario de interacción social las redes de conocimiento son un apoyo intelectual de alto nivel para quienes establecen en las herramientas de comunicación un estilo de aprendizaje continuo, no obstante antes de llegar a esa autopista el adulto mayor deberá recorrer ciertos senderos alternos que le permitan tener un desenvolvimiento adecuado, este terreno esta ganado con los docentes en general, para citar un grupo de usuarios, no obstante he aquí que las herramientas y métodos que se introduzcan en esta contribución tecnológica se expanden a otros grupos de adultos mayores (desde abogados, ingenieros, arquitectos, maestros en arte, psicólogos, pasando por personas jubiladas de entidades diferentes a las del sector educación, hasta personas de gran variedad de experiencias en un sector laboral amplio), por consiguiente se opta por proponer una metodología híbrida (Jiménez-Hernández \& Orantes-Jiménez, 2012), es decir, pueden ser una combinación de varias metodologías. Según el estudio titulado "Metodología Híbrida para Desarrollo de Software en México" se realizó teniendo en cuenta las necesidades actuales de las empresas mexicanas dedicadas al desarrollo de software. Esta metodología combina algunas prácticas existentes dentro de las metodologías RUP, XP y Scrum que son las más utilizadas en el mundo por los desarrolladores de software (Jiménez-Hernández \& Orantes-Jiménez, 2012).

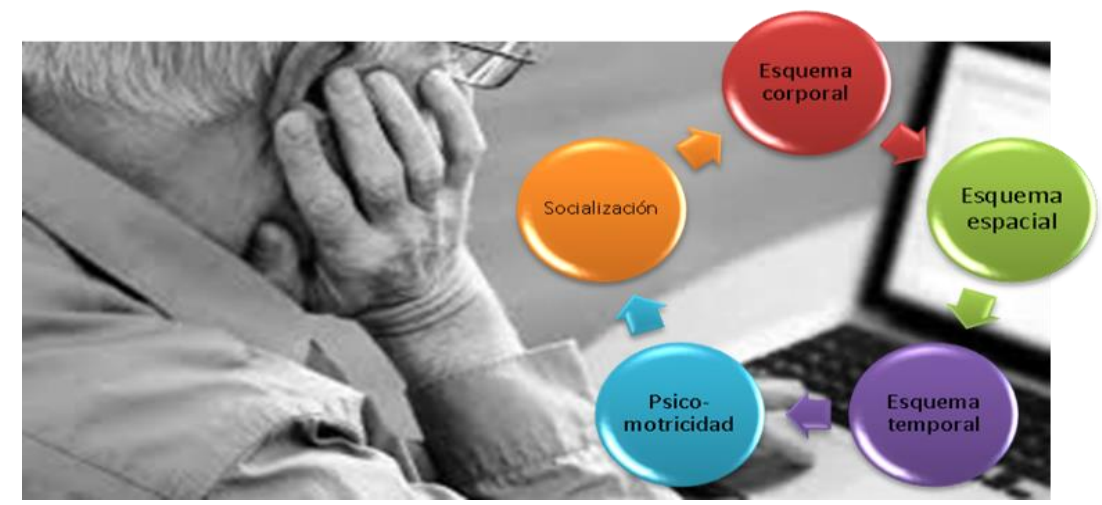

Figura 4. Adulto mayor

Fuente: (Fundación Carlos Slim, 2014)

Con base al anterior análisis del diseño de una contribución tecnológica para adultos mayores, se propone además establecer la conceptualización específica de esta metodología en distintos aspectos que sirva de guía para el desarrollador. Esta metodología obedece a que el desarrollador cuente con cuatro fases a saber:

Planteamiento. Conocimiento que permite viabilizar mediante entrevista de los grupos focales la realidad de la calidad de vida, así como implicaciones físicas, sociales, e intelectuales de los participantes que estén ligados al propósito de contribuir a través del uso de la tecnología para apoyar a la integración social del adulto mayor.

Análisis e interpretación del diseño. Con base en los insumos colectados de las respuestas de los adultos mayores participantes, aquí entra a colación el ejercicio de atender a ese sistema de afirmaciones para establecer relaciones (implicación, causalidad, correlación, probabilidad, entre otros) entre conceptos. Este podría ser un aporte para aseverar la participación de los investigadores en una mejora para el fenómeno de transformación social que con el envejecimiento se está incrementando en todos los países del mundo.

La construcción de la teoría. Se tiene en cuenta lo conceptual, en este caso son amplios conjuntos de conceptos y relaciones entre ellos, categorizaciones y el componente experimental que sugiere la diversidad.

La propuesta de metodología de software: Planificación, diseño, construcción, implementación, pruebas, realimentación ,y evaluación.

Ciertamente, las declaraciones pueden diferir según los niveles de verificación, generalidad, productividad (Swanson \& Chermack, 2013) y, por lo tanto, una teoría evoluciona y se vuelve más compleja a medida que se admite la heurística. La adultez, la experiencia, el conocimiento aplicado, el mantenimiento de las habilidades cognitivas en el tiempo, la complejidad del pensamiento, el enorme panorama de intereses que mueve al adulto mayor a participar progresivamente en la era de información y del conocimiento, permitirá a futuro la construcción de teorías, puede incluso convertirse en un modelo de trabajo y pensamiento para los científicos, es decir, un paradigma. 


\section{Resultados esperados}

Con la revisión sistemática de fuentes de información como: revistas científicas, bases de datos y repositorios digitales con autoridad académica científica, se logrará la construcción de teoría en ciencia aplicada en fase conceptual y en fase operacional, para finalmente generar, validar y evaluar estrategias tecnológicas basadas en entornos colaborativos, lúdicos y ubicuos, que permitan potenciar las habilidades cognitivas y psicomotoras de los adultos mayores.

Para el desarrollo de las estrategias es necesario acoger el pensamiento heurístico frente a las necesidades del usuario, no necesariamente algorítmico, se deberá siempre tener en cuenta el orden, la estructura, los conceptos y las categorías. Si usamos una imagen construida a partir de la Taxonomía de Bloom (ver Figura 5), se puede observar como el ser humano desarrolla una habilidad que hasta la adultez se manifiesta: esa habilidad es ordenar, y es fundamental para los propósitos del producto digital a crear.

Las caracterizaciones que se van a generar son para personas con determinados niveles de conocimiento. El punto de partida puede ser por el contexto en el cual el usuario se desarrolla. Selecciona respuestas a preguntas previamente estructuradas (adquirirlas por encuestas, entrevistas de profundidad, grupos focales, estudios documentales de estadísticas sobre el tema), en torno a:

- Meditación reflexiva

- Estados del conocimiento de un tema

- Renovación del pensamiento

- Contexto situación actual

- Búsqueda heurística

- Análisis de las dificultades para encontrar un conjunto de soluciones

- Resolución de problemas en escenarios reales

- Aportes de sus experiencias para las generaciones más jóvenes

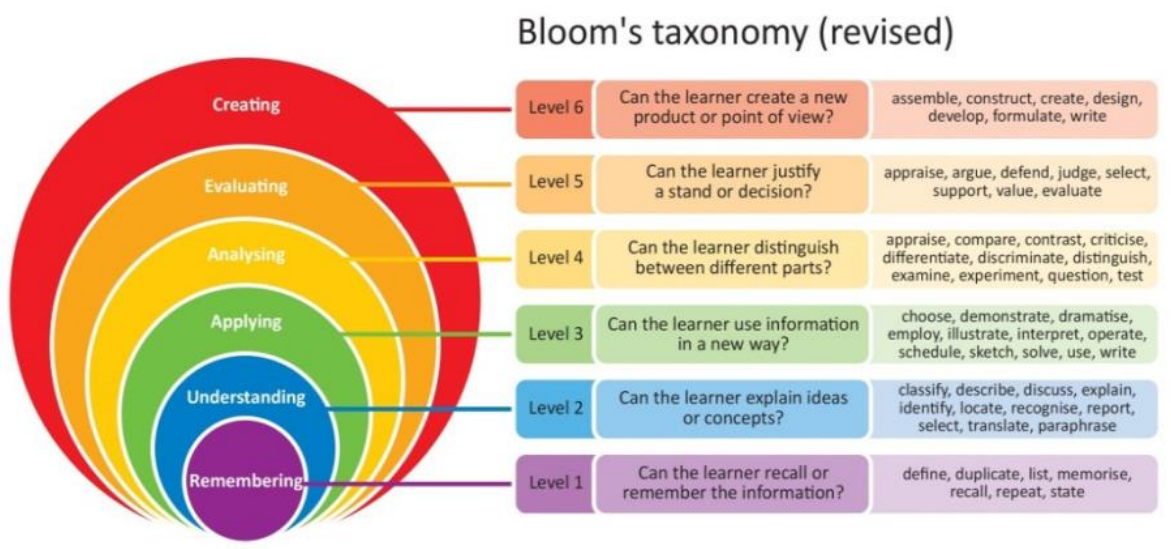

Figura 5. Taxonomía Bloom

Fuente: (McNullty, 2017)

El desarrollo de la investigación requiere de un proceso lógico, en el que se integran actores, saberes, técnicas, herramientas y actividades (ver Figura 6).

\section{Conclusiones}

El adulto mayor con el envejecimiento disminuye algunas de sus habilidades, sin embargo, el conocimiento que posee es una riqueza que hay que canalizar a través de esta contribución tecnológica, para que alcance a las generaciones jóvenes. El paso del tiempo requiere mantener un ritmo de "entrenamiento" intelectual que permita al adulto mayor interactuar por más tiempo con el conocimiento, y extienda el uso de sus habilidades. 
El propósito es que la contribución tecnológica basada en metodologías híbridas logre atraer al adulto mayor, y con ello, lo adentre en un proceso para la renovación en el pensamiento frente a la importancia de intencionar el aprendizaje continuo, en tanto se eleve la comprensión de sus condiciones, y en consecuencia supla sus necesidades específicas de interacción social, las cuales repercuten directamente en su calidad de vida.

Esta contribución tecnológica podrá ser personalizada de manera individual y puede crecer en complejidad, en tanto identifique claramente lo que el adulto mayor requiere. De todos modos, la transformación social manifiesta es una realidad en los diferentes países, por lo que no se podría albergar una sola metodología, se debe recurrir a la heurística, a procesos de meta cognición y organización de metadatos, consecuentemente con la diversidad de intereses que pueden darse en la síntesis de la recolección de la información sobre adultos mayores.

Las metodologías híbridas aumentan la complejidad del software en términos de bases de conocimiento, pero una vez estudiadas y claramente entretejidas facilitaran enormemente la producción de esta contribución tecnológica en el orden de tiempo, porque es significativa la cantidad de personas mayores en el mundo, y en costos, puesto que, si el tiempo de dedicación es menor y al usar recursos como los marcos de trabajo, se facilita la construcción de software.

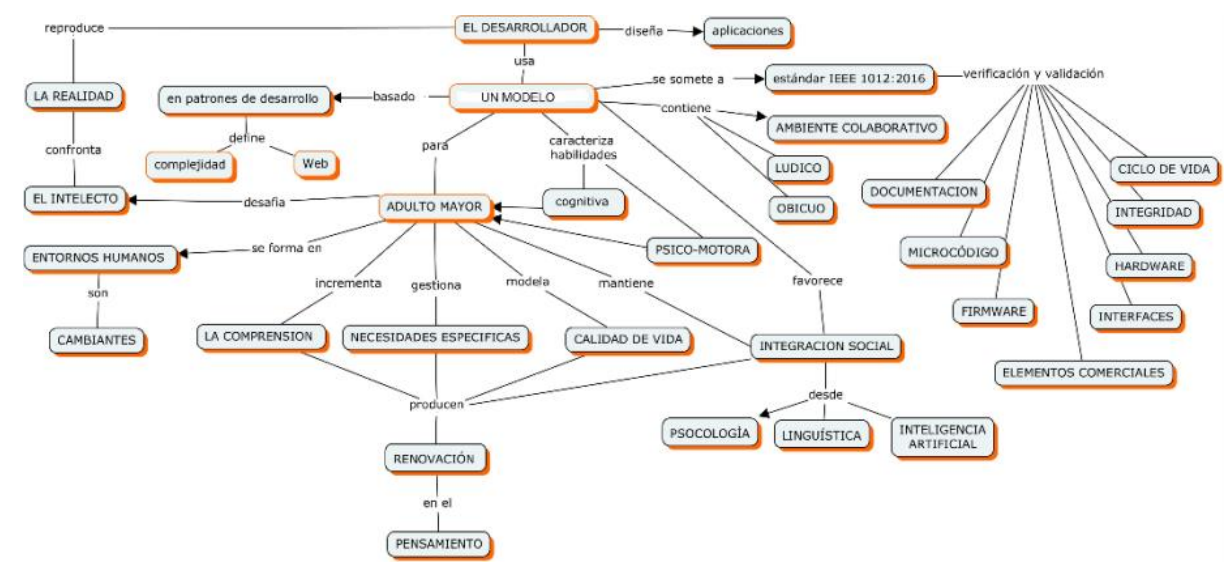

Figura 6. Esquema general que abarca la propuesta

\section{Declaración de conflicto de intereses}

Los autores declaran no tener conflicto de intereses con respecto a la investigación, autoría o publicación de este artículo.

\section{Referencias}

Alonso Jiménez, R., \& Moros García, M. T. (2011). Intervención Psicomotriz en Personas Mayores. TOG (A Coruña), 8(14), 13. https://dialnet.unirioja.es/descarga/articulo/3751050.pdf

Beaudry Bellefeuille, I. (2013). El enfoque de la integración sensorial de la doctora Ayres. TOG (A Coruña), 10(17), 11. http://revistatog.com/num17/pdfs/historia1.pdf

Comisión Económica para América Latina y el Caribe. (2018). Envejecimiento, personas mayores y Agenda 2030 para el Desarrollo Sostenible: perspectiva regional y de derechos humanos (S. Henchuan (ed.)). Naciones Unidas. https://repositorio.cepal.org/handle/11362/44369

Correia, L., Costa, N., \& Pereira, A. (2014). Fighting Elders' Social and Technological Exclusion: The TV Based Approach. En C. Ramos, P. Novais, C. E. Nihan, \& J. M. Corchado Rodríguez (Eds.), Ambient IntelligenceSoftware and Applicationes (pp. 209-216). Springer, Cham. https://doi.org/10.1007/978-3-319-07596-9 23

del Popolo, F. (2001). Características sociodemográficas y socioeconómicas de las personas de edad en América Latina. CEPAL, Población y Desarrollo. https://repositorio.cepal.org/handle/11362/7154

Fundación Carlos Slim. (2014). Adulto mayor. https:/www.clikisalud.net/wp-content/uploads/2014/01/adultomayor.jpg

Galarza Vásquez, K. (2012). Envejecimiento activo, mejor vida en la tercera edad. 
https://www.saludymedicinas.com.mx/mujer/menopausia/envejecimiento-activo-calidad-de-vida-en-la-terceraedad

Jiménez-Hernández, E. M., \& Orantes-Jiménez, S. D. (2012). Metodología Híbrida para Desarrollo de Software en México. Memorias de la Segunda Conferencia Iberoamericana de Complejidad, Informática y Cibernética (CICIC 2012), 5. http://www.iiis.org/CDs2012/CD2012IMC/CICIC 2012/PapersPdf/CB153YB.pdf

Klimova, B., \& Poulova, P. (2018). Older People and Technology Acceptance. En J. Zhou \& G. Salvendy (Eds.), Human Aspects of IT for the Aged Population. Acceptance, Communication and Participation. ITAP 2018. Lecture Notes in Computer Science (pp. 85-94). Springer, Cham. https://doi.org/10.1007/978-3-319-92034-4_7

McNullty, N. (2017). Bloom's Digital Taxonomy. Niall McNullty.com. https://www.niallmcnulty.com/2017/11/blooms-digital-taxonomy/

Menéndez Montañés, M. C., \& Brochier Kist, R. B. (2011). La actividad física y la psicomotricidad en las personas mayores: sus contribuciones para el envejecimiento activo, saludable y satisfactorio. Textos \& Contextos (Porto Alegre), 10(1), 179-192. https://www.redalyc.org/pdf/3215/321527168014.pdf

Naciones Unidas. (2002). Declaración Política y Plan de Acción Internacional de Madrid sobre el Envejecimiento. Departamento de Información Pública. https://social.un.org/ageing-working-group/documents/mipaa-sp.pdf

Nazareno Boarini, M., Cerdá, E. P., \& Rocha, S. (2006). La educación de los adultos mayores en TICs. Nuevas Competencias para la Sociedad de Hoy. Revista Iberoamericana de Tecnología en Educación y Educación en Tecnología, 1(1), 7. http://teyet-revista.info.unlp.edu.ar/TEyET/article/view/174

Ramscar, M., Hendrix, P., Shaoul, C., Milin, P., \& Baayen, H. (2014). The Myth of Cognitive Decline: Non-Linear Dynamics of Lifelong Learning. Topics in Cognitive Science, 6(1), 5-42. https://doi.org/10.1111/tops.12078

Sevilla Caro, M., Salgado Soto, M. del C., \& Osuna Millán, N. del C. (2015). Envejecimiento activo. Las TIC en la vida del adulto mayor. Revista Iberoamericana para la Investigación y el Desarrollo Educativo, 6(11), 15. https://www.redalyc.org/pdf/4981/498150319034.pdf

Shore, L., Power, V., de Eyto, A., \& O’Sullivan, L. (2018). Technology Acceptance and User-Centred Design of Assistive Exoskeletons for Older Adults: A Commentary. Robotics, 7(1), 3. https://doi.org/10.3390/robotics7010003

Swanson, R. A., \& Chermack, T. J. (2013). Theory building in applied disciplines. Berrett-Koehler Publishers.

United Nations. (2015). World Population 2015. Department of Economic and Social Affairs. Population Division. https://population.un.org/wpp/Publications/Files/World_Population_2015_Wallchart.pdf

United Nations. (2019). Perspectivas de la Población Mundial 2019: Aspectos Destacados. United Nations Department of Public Information. https://population.un.org/wpp/Publications/Files/WPP2019 PressRelease ES.pdf

Valencia Vásquez, J., Morante Ortiz, P., \& Soto Añari, M. F. (2011). Velocidad de procesamiento y memoria de trabajo en adultos mayores: Implicancias para el envejecimiento cognitivo normal y patológico. Revista de Psicología, 1,11-26. http://35.161.26.73/index.php/psicologia/article/view/1 